\title{
Mediennutzung und -konsum bei Kindern und Jugendlichen
}

\author{
Stellungnahme der Österreichischen Gesellschaft für Kinder- \\ und Jugendpsychiatrie (ÖGKJP) und der Österreichischen \\ Gesellschaft für Kinder- und Jugendheilkunde (ÖGKJ)
}

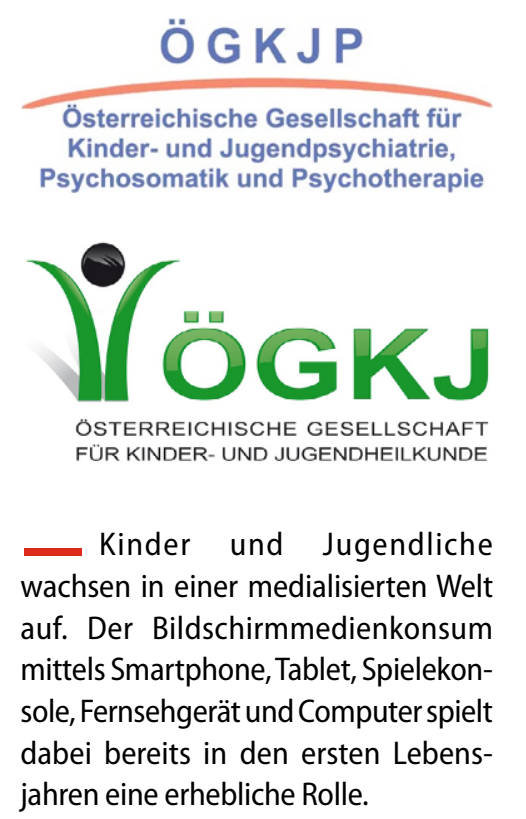

\section{Aktuelle Daten}

Aktuelle Studienergebnisse verweisen auf Zusammenhänge zwischen Entwicklungsauffälligkeiten und der Nutzungsdauer digitaler Medien bei (Klein-) Kindern und Jugendlichen. So finden sich über alle Altersgruppen hinweg gesundheitsrelevante Zusammenhänge zwischen dem digitalen Medienkonsum (Nutzungsdauer und dysregulierte Internetnutzung) und Entwicklungsauffälligkeiten wie Sprachentwicklungsstörungen, motorischer Hyperaktivität, Konzentrationsstörungen, Körpergewichtszunahme und Kontrollverlust bei Mediennutzung [1].

Je mehr Zeit Vorschulkinder täglich vor dem Bildschirm verbringen, umso häufiger leiden sie unter Schlafproblemen, wie Ein- und Durchschlafprob- lemen, schlechterer Schlafqualität oder Tagesmüdigkeit [1, 2, 3,4]. Ebenso geht ein höherer Bildschirmmedienkonsum mit vermehrt aggressiven Verhaltensweisen und Aufmerksamkeitsproblemen sowie Hyperaktivität im Kindergarten- und Grundschulalter einher $[1,2,4]$. Längere Bildschirmzeiten sind zudem mit höheren BMI-Werten. [1, 2] und einer deutlichen Zunahme an Kurzsichtigkeit bei Kindern [5, 6] assoziiert.

\section{Altersgerechte Regeln}

Generell gilt: Je jünger das Kind, desto wichtiger die bildschirmfreie Zeit [1]. Ebenso fordert die American Academy of Pediatrics eine Limitation der Bildschirmzeit für Kinder bis zum sechsten Lebensjahr auf max. eine Stunde pro Tag und spricht sich gegen jegliche Bildschirmzeit bei Kindern unter zwei Jahren aus [7].

Erste Langzeitstudien [8] verweisen bei hohem Medienkonsum von 4-Jährigen auf ein schlechteres Emotionsverständnis mit sechs Jahren. Klare Regeln zur Dauer der Mediennutzung und eine inhaltliche Begleitung dieser erscheinen als essentiell.

\section{WHO-Empfehlungen}

Die WHO-Empfehlungen für Kinder entsprechen der Datenlage - so belaufen sich die Empfehlungen für Säuglinge (bis zum vollendeten 12 . Lebensmonat) auf 0 Minuten Bildschirmzeit, bei Kindern bis zu einem Alter von zwei Jahren sollte die sitzende Bildschirmzeit nicht mehr als 60 Minuten in Begleitung einer Bezugs- person betragen. Bei Kleinkindern im Alter von drei bis vier Jahren wird nicht mehr als 60 Minuten Bildschirmzeit pro Tag empfohlen.

Der Unterschied zwischen den 2-Jährigen und den 3-4-Jährigen liegt darin, dass bei unter 2-Jährigen eine erwachsene Bezugsperson ständig anwesend sein soll.

Altersgerechte Inhalte, eine Begrenzung (z. B.; "bildschirmfreie“ Kinderzimmer) und Begleitung des Medienkonsums durch Erwachsene und insbesondere viel Bewegung und Erfahrungen im realen Leben erscheinen als maßgeblich für eine gesunde Entwicklung.

\section{Empfehlungen der ÖGKJ}

Die Österreichische Gesellschaft für Kinder- und Jugendheilkunde hat im Jahr 2017 ähnliche Empfehlungen herausgegeben [9].

In einer Pressemeldung in der „Kleinen Zeitung" vom 30.04.2021 wird nunmehr berichtet: Eine Gruppe italienischer Parlamentarier hat der Abgeordnetenkammer einen Gesetzesentwurf vorgelegt, um bei Kindern unter zwölf Jahren die Nutzung von Smartphones und Tablets stark einzuschränken. Eltern, die sich nicht an die Vorschriften halten, müssen demnach mit Geldstrafen zwischen 300 und $1500 €$ rechnen. Laut dem Vorschlag soll in den ersten drei Lebensjahren des Kindes ein absolutes Verbot der Nutzung elektronischer Geräte gelten.

Wenn auch inhaltlich nachvollziehbar, ist eine Regulierung der Bild- 
schirmzeiten mittels Strafandrohungen an die Eltern unserer Meinung nach nicht zielführend. Kinder und Jugendliche sollen vielmehr dabei begleitet und unterstützt werden, gesunde Mediennutzungsgewohnheiten zu etablieren (Abschalten von Medien 60 Minuten vor dem zu Bett gehen, kein Medienkonsum während des Essens, kein Medienkonsum zur Emotionsregulation) [10].

Dazu bedarf es Präventions- und Interventionsmaßnahmen für Eltern und Kinder aller Bevölkerungsgruppen (insbesondere auch für sozial benachteiligte Familien).

"Klassische" Beratung erscheint dafür allerdings nicht ausreichend, erforderlich sind wahrscheinlich innovative Ideen und Angebote. Denkbar wären z. B. Handy-Gratisverträge für Kinder bzw. ein "Kinder-Billigsttarif", der z. B. mit einer zeitlichen Limitierung von 60 Minuten proTag verknüpft ist. Eine weitere Möglichkeit wäre eine „Kinder-App" mit Zeitzähler, eingeblendeterWarnung und automatischer Abschaltung der Internetnutzung nach einer vorgegebenen Zeit.

\section{Präventionsmaßnahmen}

Technisch müssten derartige Präventionsmaßnahmen relativ leicht umsetzbar sein, wichtig wäre es, die Mobilfunkbetreiber für diese Art des „Kinderschutzes" zu gewinnen.

\section{Weitere Informationen:}

Fragen an die ÖGKJ oder an die ÖGKJP? www.paediatrie.at; oegkj@studie12.co.at www.oegkjp.at; office@oegkjp.at
Wir stehen für Fragen jederzeit zur Verfügung und verbleiben mit freundlichen Grüßen,

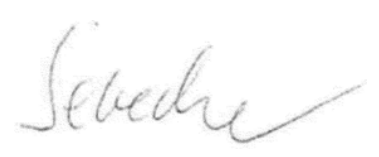

Univ.-Prof. Dr. Kathrin Sevecke Präsidentin der ÖGKJP / in Vertretung des Präsidiums

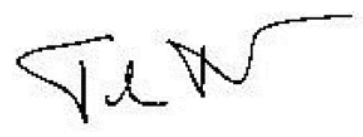

Univ.-Prof. Dr. Paul Plener, MHBA Vizepräsident der ÖGKJP

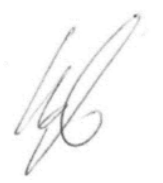

Dr. Klaus Kapelari

Leiter Kinderschutzgruppe Klinik Innsbruck

$$
\text { Salo } \int l d x-B u i n
$$

Priv.-Doz. Dr. Sabine Scholl-Bürgi ÖGKJ Erste Sekretärin

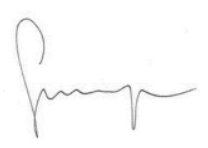

Assoz.-Prof. PD Dr. Volker Strenger Leiter / AG Infektiologie der ÖGKJ

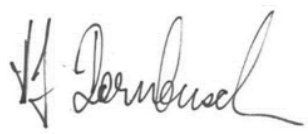

Priv.-Doz. Dr. Hans Jürgen Dornbusch Leiter / Impfreferat der ÖGKJ

\section{Damile Karall}

A.Univ.-Prof.Dr.Daniela Karall

Präsidentin der ÖGKJ

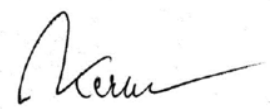

Univ.-Prof. Dr. Reinhold Kerbl

Generalsekretär der ÖGKJ

\section{Literatur}

1. Büsching U, Riedel R (2017) BLIKK-Medien: Kinder und Jugendliche im Umgang mit elektronischen Medien. https://www. drogenbeauftragte.de/fileadmin/Dateien/5_Publikationen/Praevention/Berichte/ Abschlussbericht_BLIKK_Medien.pdf

2. Bernath J, Waller G, Meidert U (2020) ADELE+ : der Medienumgang von Kindern im Vorschulalter (4-6 Jahre) - Chancen und Risiken für die Gesundheit. Obsan Bericht; 03. Neuchâtel: Schweizerisches Gesundheitsobservatorium (Obsan) https:// doi.org/10.21256/zhaw-20447. ISBN 978-2940670-02-4

3. Genuneit J, Brockmann PE, Schlarb AA, Rothenbacher D (2018) Media consumption and sleep quality in early childhood: results from the UIm SPATZ Health Study. Sleep Med 45:7-10. https://doi.org/10.1016/j. sleep.2017.10.013

4. Maass EE, Hahlweg K, Heinrichs N, Kuschel A, Naumann S, Bertram H, Staender D, Doepfner M (2010) Sozioökonomischer Status, mütterliches Erziehungsverhalten, erhöhter Medienkonsum und die Sprachund Rechenfertigkeiten von Kindergartenkindern. Psychol Erziehung Unterr - Z Forsch Prax 57(1):46-61. https://doi.org/10.2378/ peu2010.art04d

5. Saxena R, Vashist P, Tandon R, Pandey RM, Bhardawaj A et al (2017) Incidence and progression of myopia and associated factors in urban school children in Delhi: The North India Myopia Study (NIM Study) Incidence and progression of myopia and associated factors in urban school children in Delhi: The North India Myopia Study (NIM Study). PLoS ONE 12(12):e189774. https:// doi.org/10.1371/journal.pone.0189774

6. Jiaxing Wang MD, Li YMD, Musch DC, Wei N, Xiaoli Q, Ding G, Li X, Li J, Linlin S, Zhang Y, Yuxian N, Xiaoyu Z, Hua N, Li S, Xuehan Q (2021) Progression of Myopia in School-Aged Children After COVID-19 Home Confinement. JAMA Ophthalmol 139(3):293-300. https://doi.org/10.1001/ jamaophthalmol.2020.6239

7. Chassiakos YLR, Radesky J, Christakis DMAM, Corinn Cross (2016) Children and Adolescents and Digital Media. Pediatrics 
138(5):e20162593. https://doi.org/10.1542/ peds.2016-2593

8. Skalická V, Wold Hygen B, Stenseng F, Berg Kårstad S, Wichstrøm L (2019) Screen time and the development of emotion understanding from age 4 to age 8: A community study. Br J Dev Psychol 37(3):427-443. https://doi.org/10.1111/bjdp.12283

11. Sauseng W, Sonnleitner A, Hofer N, Pansy J, Kiechl-Kohlendorfer U, Weiss S, Kenzian Kerbl HR (2017) Empfehlungen zur Regulierung von Bildschirmzeiten im Kindes- und Jugendalter Konsensuspapier der Arbeitsgruppe Schlafmedizin und Schlafforschung der Österreichischen Gesellschaft für Kinder- und Jugendheilkunde. Monatsschr Kinderheilkd 165:254-256. https://doi.org/10.1007/s00112-016-0201-0

10. American Academy of Child and Adolescent Psychiatry, 2020

Hinweis des Verlags. Der Verlag bleibt in Hinblick auf geografische Zuordnungen und Gebietsbezeichnungen in veröffentlichten Karten und Institutsadressen neutral.

Paediatr. Paedolog. 2021 · 56:250-252 https://doi.org/10.1007/s00608-

021-00935-0

(c) Springer-Verlag GmbH Austria, ein Teil von Springer Nature 2021

Hier steht eine Anzeige.

\section{Springer}

